\title{
MODIFIED GPSR FOR FAULT TOLERANT AND ENERGY EFFICIENCY IN MOBILE AD HOC NETWORK
}

\author{
Jyotsana Jaiswal and Pabitra Mohan Khilar \\ Department of Computer Science and Engineering, National Institute of Technology \\ Rourkela, India \\ jyotsanaa.jaiswal@gmail.com \\ pmkhilar@itrkl.ac.in
}

\begin{abstract}
Routing in wireless network is a key research area which establishes path between source and destination node pairs. In this paper, we have designed and evaluated an Energy-efficient and Fault tolerant Greedy Perimeter Stateless Routing (EFGPSR) protocol for wireless ad hoc network. The proposed protocol is divided into four phases: Fault testing phase, Planarization phase, Energy efficient greedy forwarding phase and Energy efficient perimeter forwarding phase. In fault testing phase, all nodes come to know about their fault free neighbours. Next is planarization phase which removes the crossing edges. Next are energy efficient greedy forwarding and perimeter forwarding phases. Both these phases try to maintain balance between the metrics to choose the next hop (i.e. distance from destination in greedy forwarding phase and minimum angle w.r.t the line connecting the forwarding node and destination in perimeter forwarding phase) and selection of node having highest energy among the neighbouring nodes to extend network lifetime. Evaluation and comparison of GPSR and EFGPSR is done through NS-2 simulator. Simulation shows that EFGPSR performs better in terms of increasing the network lifetime, successful packet delivery ratio with insignificant increase in number of hop count.
\end{abstract}

\section{KEYWORDS}

Wireless network, geographic routing, fault tolerance, GPSR, planarization

\section{INTRODUCTION}

In recent years, wireless and mobile communication technologies have grown rapidly. As wireless network evolves there is a trend towards decentralized, deployable and self-organizing networks. Routing plays a vital role in establishing communication links between various nodes. Since last few years, geographic routing (GR) protocols, also known as geo-routing or position based routing for wireless networks has gained a significant attention. The idea behind GR protocol is that the source node sends a packet to destination node using the geographic location of destination, instead of using network address. The essential requirements of GR are a) each node should be capable of determining its own location (geographic coordinates) and b) source should be aware of the location of destination. The main advantage of GR over traditional routing protocol is that each node requires maintaining only location of itself and its neighbours for its functioning. However, in traditional routing protocols for wireless networks (e.g. AODV [1], DSDV [2]), nodes usually have to keep significant amount of routing information. Greedy Perimeter Stateless Routing (GPSR) [3] is a well-known and most commonly used position-based 
Computer Science \& Engineering: An International Journal (CSEIJ), Vol.1, No.5, December 2011

routing protocol for wireless networks. In GPSR, source includes the location information of destination in the header of every packet. If the destination is not directly reachable, the source starts with greedy forwarding, i.e., the source node forwards the data packet to the neighbour that is closest to the destination in the coordinate space. Such greedy forwarding is repeated at the intermediate node, until the destination is reached. However, GPSR itself suffers from few drawbacks. Firstly, greedy forwarding over geographic coordinates may not be optimal due to unawareness of connectivity information of the network. Secondly, packet may get stuck in local minimum condition (a forwarding node could not find a neighbour that lies closer to the destination than itself) for sparse networks. To deal with above stated local minimum problem, the nodes switch to perimeter forwarding. In perimeter forwarding, the packet is forwarded to that neighbouring node which comes first in a planar sub graph of the network, when the line connecting the forwarding node and destination is rotated in the counter clockwise direction. The location of forwarding node where perimeter forwarding starts is recorded in the header of the data packet. Greedy forwarding is resumed when the data packet reaches a forwarding node which can find a neighbour node whose distance is smaller than the distance between the destination node and the node at which perimeter forwarding begun. The probability of finding a route between source destination node pairs is very high.

Most existing designs of wireless network routing protocols are based on the assumption that every node in the network is fault free. However, such assumption usually does not hold in realistic environments. Various kinds of faults such as crash faults, transient faults, and byzantine faults can occur in a node. The occurrence of faults affects the routing process therefore fault tolerance is of increasing importance in applications where it is essential to maintain efficient routing. In routing, fault tolerance helps in controlling the overhead which is there due to the faulty node and thus helps in considering the reliable routes. Many fault tolerance routing protocols have been proposed in wireless network. In Wireless Sensor Network (WSN), fault tolerance mechanism in routing protocol has been classified into two schemes, retransmission and replication. The fault tolerant routing problem in MANET is not explored to the fullest. Wireless network comprises of large number of energy constraint node, some node may run out of energy and die and shorten the lifetime of network. Therefore, many routing protocols in MANET and WSN have been proposed with the aim to reduce energy consumption [4],[5],[6],[7]. GPER [8] protocol was proposed to provide power efficient geographic routing in WSN. Traditionally, greedy forwarding and perimeter forwarding consider only single metrics i.e. minimum distance from destination and minimum angle in counter clockwise direction with respect to the line connecting the forwarding node and destination. These parameters do not take into account the energy conservation for evaluating the routing performance. In this paper, energy efficient and fault tolerant routing algorithm is proposed. The rest of the paper is organized as follows: The system models are described in Section 2. The energy efficient and fault tolerant GPSR routing protocol is presented in Section 3. Section 4 focuses on the simulation result and comparison with other existing protocol. Finally, Section 5 concludes the paper and lists the future works.

\section{Preliminaries}

\subsection{System, Fault and Energy Model}

\subsubsection{System Model}

The system is composed of homogeneous $\mathrm{n}$ mobile nodes, each having a unique id. All nodes have similar computing, storage resources and identical communication range. A set of nodes with circular radio range $r$, can be seen as a graph: each node is a vertex, and edge $(n, m)$ exists 
Computer Science \& Engineering: An International Journal (CSEIJ), Vol.1, No.5, December 2011

between nodes $n$ and $m$ if the distance between $\mathrm{n}$ and $\mathrm{m}, d(n, m) \leq r$. Source node knows the location of final destination.

\subsubsection{Fault Model}

Wireless network consists of many nodes which may be either fault free or faulty. The following assumptions have been made about faulty nodes:

1. No new faults occur during the execution of the routing protocol i.e. faults are permanent (a faulty node remains faulty until it is repaired and/or replaced).

2. Faulty nodes can be either hard faulty or soft faulty.

Nodes become hard faulty due to two main reasons. First, a node may be damaged during deployment or immediately after that. Second, because of depletion of battery power. When a node becomes hard faulty it does not participate in any further communication. Soft faulty nodes are more subtle than hard faulty nodes, since a soft-faulted node continues to communicate with the other node in the system although with altered specifications i.e., the faulty nodes may produce some random results instead of expected results. In this work, both hard-faulted and softfaulted nodes have been considered. The proposed routing protocol applies the following testing model given in Table 1 to check whether the node is faulty or fault free. The testing model uses the test task to check the validity of the node. The test task is to find out two's complement of five bit number. Tester node knows the result of the test task. Tester node broadcast the test task along with its id and location information. On receiving the test task, all the neighbouring nodes unicast the result of the test task along with their id and location information. As shown in the table of test model, five cases are observed. First case, the status of the tester node and tested node are both fault free then test result is zero. This means that there is match between the expected result of the tester node and the actual result returned by the tested node. Second case, the tester node is fault free and the tested node is soft faulty then test result is 1 . This means there is a mismatch between the expected result and actual result. Third case, the tester node is fault free or soft faulty and the tested node is hard faulty then test result is NULL because a hard faulty node can receive a beacon test message but cannot send reply to it. Fourth case, the tester node is soft faulty and tested node is soft faulty or fault free then test result is 1 . Here also there is mismatch between the expected result and actual result. Fifth case, the tester node is hard faulty and tested node is also faulty (soft or hard) or fault free then test result is NULL because a hard faulty node cannot send a beacon test message. When the test result is 0 , the node is considered as fault free, otherwise faulty. The test model given in Table 1 is used to select fault free nodes while establishing the paths between the nodes pair.

Table 1. Test model

\begin{tabular}{|c|c|c|}
\hline $\begin{array}{c}\text { Status of } \\
\text { Tester node }\end{array}$ & $\begin{array}{c}\text { Status of } \\
\text { Tested node }\end{array}$ & $\begin{array}{c}\text { Test } \\
\text { Result }\end{array}$ \\
\hline Fault free & Fault free & 0 \\
\hline Fault free & Soft faulty & 1 \\
\hline Fault free & Hard faulty & NULL \\
\hline Soft faulty & Hard faulty & NULL \\
\hline Soft faulty & Soft faulty & 1 \\
\hline Soft faulty & Fault free & 1 \\
\hline Hard faulty & Soft faulty & NULL \\
\hline Hard faulty & Hard faulty & NULL \\
\hline Hard faulty & Fault free & NULL \\
\hline
\end{tabular}




\subsubsection{Energy Model}

In wireless network energy consumption at each node can be due to transmission and reception of message. The energy model proposed by Heinzelman et al [6] to transmit an n-bit message over a distance $\mathrm{d}$ or receive an n-bit message are as follows:

$$
\begin{array}{r}
E_{T X}(m, d)=E_{T X}-\text { elec }(m)+E_{T X}-{ }_{\text {amp }}(m, d) \\
=m * E_{\text {elec }}+m * E_{\text {amp }} * d^{2} \\
E_{R X}(m)=E_{R X}-\text { elec }(m)=m * E_{\text {elec }}
\end{array}
$$

Table 2 gives related parameters and their definitions.

Table 2. Radio parameters

\begin{tabular}{|c|l|c|}
\hline Parameter & \multicolumn{1}{|c|}{ Definition } & Unit \\
\hline$E_{\text {elec }}$ & $\begin{array}{l}\text { Energy dissipation rate } \\
\text { to run the radio }\end{array}$ & $50 \mathrm{~nJ} / \mathrm{bit}$ \\
\hline$E_{\text {amp }}$ & $\begin{array}{l}\text { Energy dissipation rate } \\
\text { to run transmit amplifier }\end{array}$ & $100 \mathrm{pJ} / \mathrm{bit} / \mathrm{m}^{2}$ \\
\hline
\end{tabular}

\section{Proposed Algorithm}

The proposed algorithm consists of four different but dependent phases, which are as discussed precisely in the following sections:

\subsection{Fault Testing Phase}

In this phase, all nodes come to know about the location information and residual energy information of their fault free neighbours at regular interval. Each tester node broadcast a beacontest message containing its own id (e.g. IP address), location information and a test task. Information regarding the test task is given in 2.1. Each tester node knows the result of the test task. On receiving the beacon-test message, all the neighbouring nodes execute the test task and unicast the reply message. The reply message contains the result of the test task, their unique Id, location information and residual energy information of the node. In this phase, tester node checks whether the neighbouring nodes present are fault free or faulty (i.e., soft or hard) by comparing the result of test task using the test model given in Table 1. Each node maintains the location information and residual energy information of all fault free neighbouring nodes in their vicinity.

\subsection{Planarization Phase}

After fault testing phase, planarization phase starts. This phase can be done reactively or proactively. In proactive version, each node begins the planarization phase immediately after the fault testing phase. In reactive version, each node begins the planarization phase when local minimum condition occurs. The main reason for using the planarization phase is that the righthand rule does not work properly on fully connected graphs with crossing edge. The right hand rule is used by perimeter forwarding phase. Each node should run the planarization algorithm in a distributed fashion. The Relative Neighbourhood Graph (RNG) and Gabriel Graph (GG) are two well-known planar graphs [7]. RNG planarization is considered in this paper. During this phase 
one important property to be taken care is that, while removing edges from the graph to reduce it to RNG must not disconnect the graph.

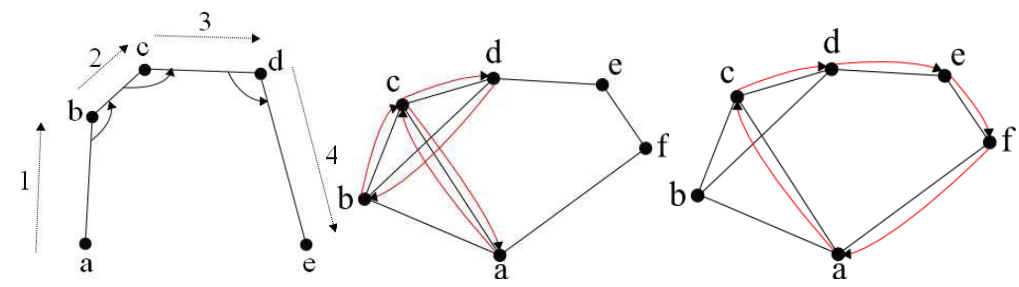

(a)

(b)

(c)

Fig. 2. (a) Right Hand Rule (RHR), (b) RHR with crossing edge, (c) RHR without crossing edge.

\subsection{Energy Efficient Greedy Forwarding Phase (EEGF)}

In this phase, a forwarding node $\mathrm{F}$ uses full graph in choosing next hop. The optimal choices of next hop consider two metrics; the minimum distance from destination $(D)$ and the energy. Algorithm 1 explains the pseudo code for the proposed energy efficient greedy forwarding used at a forwarding node. In traditional GPSR, the distance between forwarding node as well as its neighbour with respect to destination is calculated. The forwarding node selects that neighbour as the next hop that lies closest to the destination. If the forwarding node $F$ could not find a neighbour node that lies closer to the destination than itself, then node switches to perimeter forwarding. However in EEGF, forwarding node first form a set of Selected-Neighbour-list $(N)$ which is a subset of the Neighbour-list $(F)$. Each node $I \in$ Selected-Neighbour-list $(N)$, if the distance between the neighbour node $I$ and destination node $D$ is less than the distance between the forwarding node $F$ and $D$. After this, Cost $(I)$ of every node $I$ Selected-Neighbour $(F)$ is calculated. Cost $(I)$ is defined as:

$$
\operatorname{Cost}(I)=\frac{\text { ResidualEnergy }(I)}{\text { Distance }(F, I)^{2}}
$$

Where, ResidualEnergy $(I)$ is the ratio of AvailableEnergy $(I)$ and InitialEnergy $(I)$ and Distance $(F, I)$ is:

$$
\operatorname{Distance}(F, I)=\frac{\operatorname{Dist}(F-D)-\operatorname{Dist}(I-D)}{\operatorname{Dist}(F-D)}
$$

The reason for calculating this cost is that energy and distance are inversely proportional to each other because from energy balance point of view, the node with more residual energy should be selected as the next hop and from distance point of view, the node which is closer to the destination should be selected as the next hop. Thus, Cost (I) balances the two metrics energy and distance and helps in finding the optimal next hop. The forwarding node selects that neighbour I $\in$ Selected-Neighbour-list $(N)$ as next hop, whose Cost $(I)$ is maximum. If the forwarding node $\mathrm{F}$ could not find a neighbour node that lies closer to the destination than itself, that is SelectedNeighbour-list $(N)$ is empty then node switches to perimeter forwarding. 
Computer Science \& Engineering: An International Journal (CSEIJ), Vol.1, No.5, December 2011

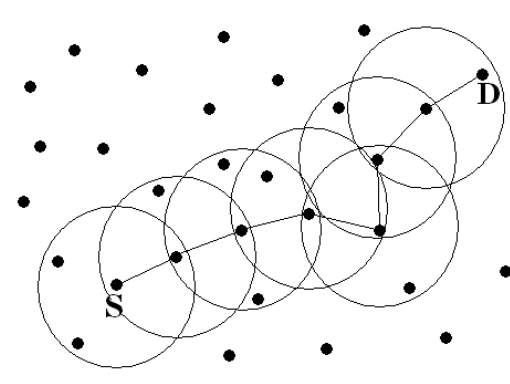

(a)

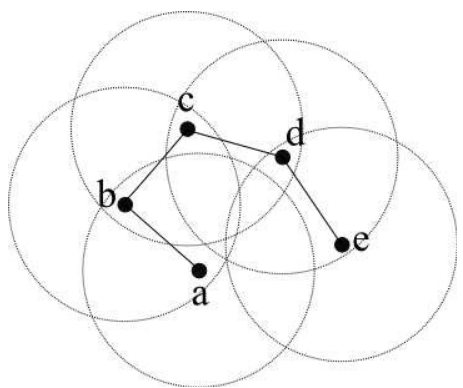

(b)

Fig. 1. (a) Greedy forwarding and (b) Local minima condition

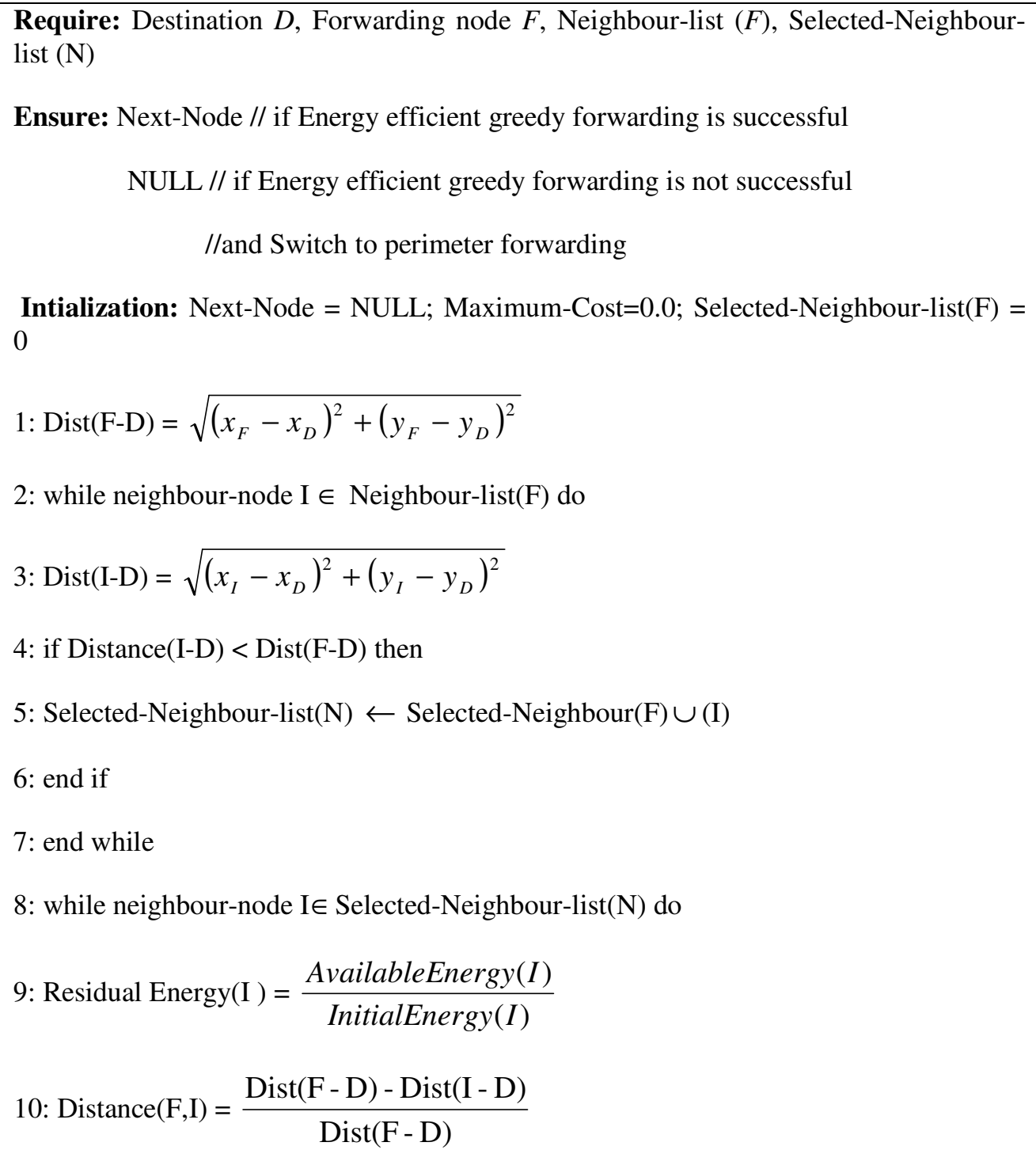


$\operatorname{Cost}(\mathrm{I})=\frac{\operatorname{Re} \text { sidualEnergy }}{\operatorname{Dist}(F, I)^{2}}$

11: if Maximum-Cost $<\operatorname{Cost}(\mathrm{I})$ then

12: Maximum-Cost $=\operatorname{Cost}(\mathrm{I})$

13: Next-Node $\leftarrow$ I

14: end if

15: end while

Algorithm 1: Energy-Efficient Greedy Forwarding Algorithm

\subsection{Energy Efficient Perimeter Forwarding}

Energy efficient perimeter forwarding uses right hand rule/left hand rule as given below: Right Hand Rule The right-hand rule to traverse a graph is shown in Figure 2 (a). This rule states that when arriving at node $b$ from node $a$, the next edge traversed is the next one sequentially counter clockwise about $b$ from edge $(a ; b)$. On graphs with edges that cross, the right-hand rule may take a degenerate tour of edges that does not trace the correct path as shown in figure 2 (b). Now on removing the crossing edges right hand rule traces the correct path (Figure 2 (c)). In this context planarization comes into picture. The left hand rule also works in a similar fashion the only difference is that the next edge traversed is in clockwise direction

Energy Efficient Perimeter Forwarding In this phase, complete EFGPSR is described which combines fault testing phase, energy efficient greedy forwarding phase and energy efficient perimeter forwarding phase. Greedy forwarding phase is executed on the full network graph whereas energy efficient perimeter forwarding phase on the planarized network graph where greedy forwarding fails. A flag is used in the packet header of EFGPSR which indicate whether

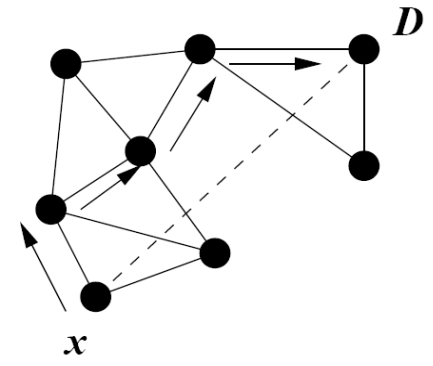

Fig. 3. Perimeter forwarding example

the packet is in greedy mode or perimeter mode. Initially, source node marks all data packets as greedy-mode. On receiving greedy-mode packet, a forwarding node uses the energy efficient greedy forwarding algorithm to find the optimal next hop. If no optimal node is found i.e. greedy forwarding fails, the node marks the packet into perimeter mode. In traditional perimeter forwarding as shown in figure 3 the packet is forwarded to that neighbour node that comes first in a planar sub graph of the network, when the line connecting the forwarding node and destination(D) is rotated in the counter clockwise direction. The location of forwarding node where perimeter forwarding starts is recorded in the header of the data packet. Greedy forwarding 
Computer Science \& Engineering: An International Journal (CSEIJ), Vol.1, No.5, December 2011

is resumed when the data packet reaches a forwarding node or a node which can find a neighbour node whose distance is smaller than the distance between the destination node and the node at which perimeter forwarding begun. However, in energy efficient perimeter forwarding, the forwarding node selects the next hop using the following algorithm:

Require: Forwarding node $F$, Neighbouring-node-list containing index, angle and energy of each node

Ensure: Next-Node

Intialization: Next-Node=NULL; Temp=0; Max=Max-energy; Min=Min-energy; Mid=Midenergy

1: Sort the Neighbouring-node-list in increasing order of angle

2: for all Neighbouring-node

3: Temp=First nodes after sorting

4: if Temp[energy] < Mid and Temp[energy] >= Max then

5: Select node index

6: break

7: end if

8: End for

Algorithm 2: Energy-Efficient Perimeter Forwarding Algorithm

\section{SimUlation RESUltS}

In this paper, the performance of GPSR and EFGPSR is evaluated based on NS-2 network simulator[12]. The network dimension used for simulation is 1000x1000 meter square. The transmission range of each node is assumed to be $250 \mathrm{~m}$. The MAC layer protocol used is IEEE 802.11. Simulation results are shown in figure 4 and figure 5.

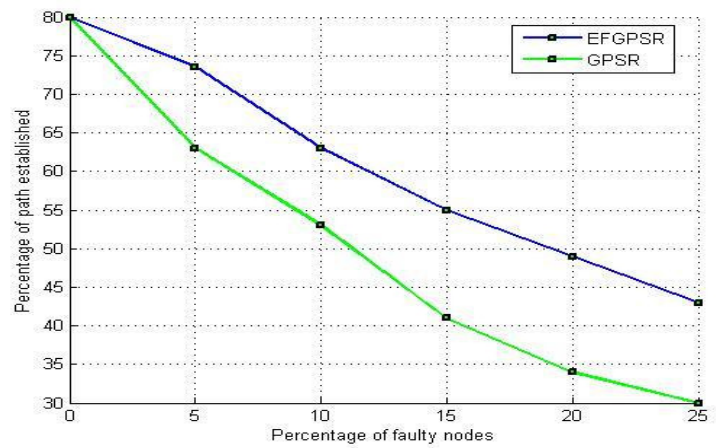

Fig. 4. 50 nodes with 30 s-d pairs with velocity of nodes $20 \mathrm{~m} / \mathrm{s}$ 


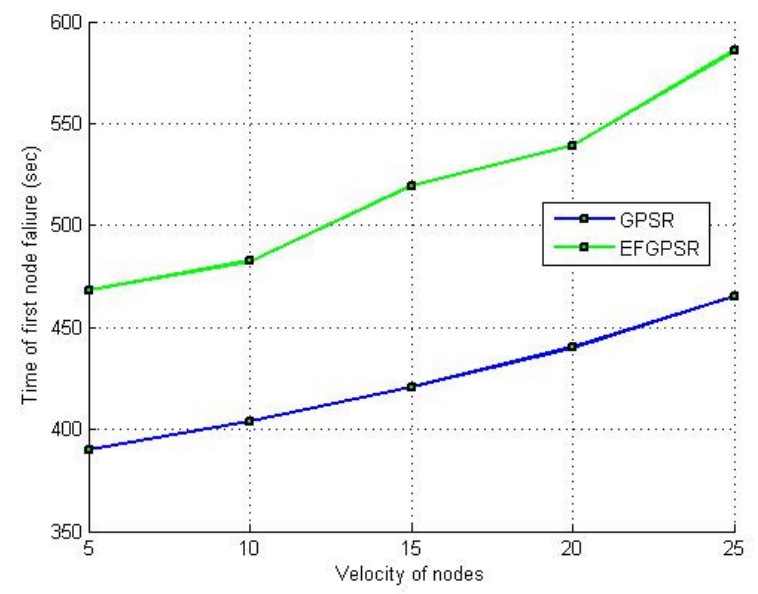

Fig. 5. 30 nodes with $15 \mathrm{~s}$-d pairs having 50\% greedy forwarding and 50\% perimeter routing and $10 \%$ node both hard and soft faults

\section{CONCLUSIONS}

In this paper, fault tolerant GPSR routing algorithm is proposed. EFGPSR and GPSR have been shown in Figure 5. And also EFGPSR try to maintain balance between the metrics to choose the next hop (i.e. distance from destination in greedy forwarding phase and minimum angle with respect to the line connecting the forwarding node and destination in perimeter forwarding phase) and selection of node having highest energy among the neighbouring node to extend network lifetime. Evaluation and comparison of GPSR and EFGPSR shows that EFGPSR performs better in terms of increasing the network lifetime, successful packet delivery ratio with insignificant increase in number of hop count.

\section{REFERENCES}

[1] Perkins, Belding-Royer \& Das, (2003) "Ad hoc On-Demand Distance Vector (AODV) Routing", RFC Editor

[2] Charles, Perkins \& Bhagwat, (1994) "Highly dynamic Destination-Sequenced Distance-Vector routing (DSDV) for mobile computers", SIGCOMM Comput. Commun. Rev. 24, pp 234-244

[3] Karp \& Kung, (2000) "GPSR: greedy perimeter stateless routing for wireless networks", In Proceedings of the 6th annual international conference on Mobile computing and networking, pp 243254

[4] Singh, Woo \& Mghavendra, (1998) "Power-Aware Routing in Mobile Ad Hoc Networks", Proceedings of the 4th annual ACM/IEEE international conference on Mobile computing and networking, pp 181-190

[5] Stojmenovic, \& Lin, (2001) "Power-Aware Localized Routing in Wireless Networks", IEEE Trans. Parallel Distrib. System, Vol. 12, No. 11, pp 1122-1133

[6] Stojmenovic, (2006) "Localized network layer protocols in wireless sensor networks based on optimizing cost over progress ratio", Network, IEEE, Vol. 20, No. 1, pp 21-27 
Computer Science \& Engineering: An International Journal (CSEIJ), Vol.1, No.5, December 2011

[7] Lee, Bhattacharjee, \& Banerjee, (2005), "Efficient geographic routing in multihop wireless networks", In Proceedings of the 6th ACM international symposium on Mobile ad hoc networking and computing (MobiHoc '05), pp 230--241

[8] Wu \& Candan, (2004) "GPER: Geographic Power Efficient Routing in Sensor Network", In Proceeding of Int'l Conference Network protocols (ICNP), pp 161-172

[9] Melodia, Pompili \& Akyildiz, (2004) "Optimal Local Topology Knowledge for Energy Efficient Geographical Routing in Sensor Network”, In Proceeding of INFOCOM

[10] Heinzelman, Chandrakasan \& Balakrishnan, (2000) "Energy-Efficient Communication Protocol for Wireless Microsensor Networks", In Proceedings of the 33rd Hawaii International Conference on System Sciences IEEE Computer Society, Vol. 8

[11] Agarwal \& Matausek, (1992) "Relative neighbourhood graphs in three dimensions", In Proceedings of the third annual ACM-SIAM symposium on discrete algorithms. Society for Industrial and Applied Mathematics, pp 58-65

[12] Kannan, Varadhan (2003) “The ns Manual (ns Notes and Documentation)”, The VINT project

\section{Authors}

Jyotsana Jaiswal received the B.E degree in Information Technology from North Maharastra University. She is currently pursuing M.Tech by Research at NIT Rourkela, India. Her research interests include routing protocol in ad hoc network, fault tolerant and energy efficieny techniques.

P. M. Khilar graduate from Mysore University in Computer Science \& Engineering in the year 1990. He received his M.Tech Degree in Computer Science from NIT, Rourkela India in the year 1999. He obtained his Ph.D degree from Indian Institute of Technology, Kharagpur, India in the year 2009. At present he is a senior faculty in the department of Computer Science and Engineering of NIT, Rourkela, India. His current research interests include parallel and distributed processing, fault tolerant computing and ad-hoc and sensor networks.
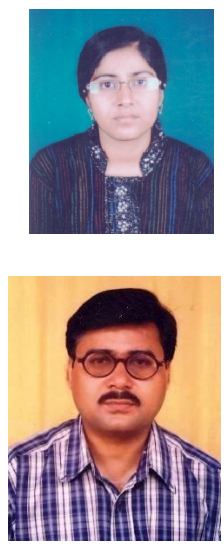